\title{
Die zwei Welten der A- und der B-Schweiz
}

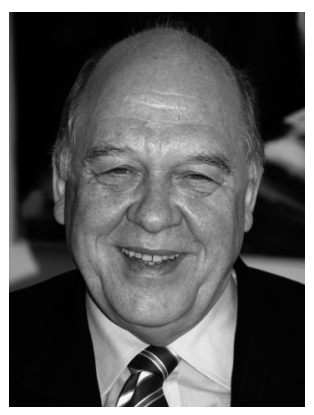

Klaus J. Stöhlker"

Die Schweizer Wirtschaft kann im Zuge der Globalisierung längst nicht mehr als Einheit gesehen werden. Vielmehr verläuft eine unsichtbare Grenze zwischen den global tätigen Konzernen und Dienstleistungsfirmen, die in der Schweiz ansässig sind, und jenen, denen das Attribut des „global players“ nicht zugesprochen werden kann. Die Schweizer Wirtschaft ist deshalb geteilt in eine A-Schweiz von etwa tausend Grossunternehmen, von Nestlé bis Glencore, die einen Haupt- oder Steuersitz im Lande haben, wozu auch die „big four"-Treuhandfirmen, die FIFA und viele Beratungsfirmen zählen, wie in eine B-Schweiz klassischer Schweizer KMU- und Gewerbebetriebe, die nur national oder regional bedeutend sind. Die Firmen der A-Schweiz werden vom Bundesrat und den kantonalen Regierungen mittels steuerlichen Begünstigungen und grossem Aufwand gesucht und gefördert. Die Unternehmen der B-Schweiz und der Schweizer Mittelstand tragen mit ihren Steuerleistungen die Erhaltung und den Ausbau der Infrastruktur des Landes.

Die Projektion falscher Bilder in den Köpfen der Menschen, wovon auch die Spitzenmanager in Politik und Wirtschaft nicht ausgenommen sind, führt häufig zu fatalen Fehlurteilen, die eine ganze Gesellschaft gefährden können. Während die mythische Schweiz dauerhaften Erfolgs und korporativer Leistung bis in das zweite Drittel des 20. Jahrhunderts hinein die Vorstellungen der Menschen beherrschte, hat seit zwei Generationen ein Prozess eingesetzt, der in der Folge der Globalisierung zu einer dauerhaften Herauslösung wachsender Teile des alten Nationalstaats geführt hat. Aus dem Nationalstaat Schweiz wird die in globale Institutionen integrierte semiautonome Schweiz. Die derzeit noch gefeierte Swissness wird im Rahmen internationaler Abkommen, wie TTIP eines ist, nicht mehr ein Qualitätsmerkmal sui generis, sondern lediglich ein Attribut unter vielen, das den Verkauf von Uhren, Milch- und Schokoladeprodukten wie Präzisionsmaschinen global zu fördern hat.

Es sind die Grosskonzerne und Dienstleistungsunternehmen, die Banken und internationalen Organisationen, die mit ihren weltumspannenden Netzen die A-Schweiz bilden. Dort gelten Spielregeln und Verhaltensprinzipien, wie sie den Unternehmen der B-Schweiz, die alleine national oder grenzüberschreitend ausgerichtet sind, weitgehend unbekannt sind. Die Unternehmen dieser Art mit Kristallisationspunkten in Basel, Zürich, Zug, Lugano und Genf haben Unternehmenskulturen entwickelt, die mit der alten Schweiz nichts mehr zu tun haben. Es sind Globalkonzerne aus aller Welt, welche die Schweiz als Standort nutzen, weil er steuerliche, geografische, strukturelle und humane Werte besitzt, die eine kritische Menge bilden, welche den Standort Schweiz für sie interessant machen. Jüngste Beispiele sind die Rohstoffhändler und die Rückversicherungen. Beide gab es schon seit über hundert Jahren im Land, aber heute bilden sie in der Schweiz die welt-

* Klaus J. Stöhlker ist Unternehmensberater für Öffentlichkeitsbildung in Zollikon/ZH. 
grössten Cluster, sei es rund um Zürich im Falle der globalen Rückversicherer oder in Zug, Lausanne und Genf im Falle der Rohstoffhändler.

Was als schweizerisch bezeichnet wird, ist längst nicht mehr unter Schweizer Kontrolle oder Führung. Besonders auffallend ist dies im Falle der beiden Grossbanken UBS und CSGroup, die ihre Wurzeln zwar in Winterthur und Zürich haben, deren Aktienmehrheit und operative Führung längst von Ausländern kontrolliert wird. Die UBS hat dem mit ihrer jüngsten Strukturanpassung Rechnung getragen, wo die Schweiz nur noch eines ihrer vier unternehmerischen Beine ist. Als ich den Verwaltungsrat einer dieser beiden Grossbanken jüngst fragte: „Was ist an Ihnen noch schweizerisch?“, sagte er: „Genügt Ihnen unser VRPräsident nicht?" de facto sind diese Konzerne in ausländischer Hand, ganz wie Nestlé, Novartis und viele andere auch.

Entscheidend ist die Tatsache, dass Bundesrat, kantonale Verwaltungen und sämtliche Regierungsparteien diesen Prozess seit fünfzig Jahren aktiv fördern. Die Konzerne haben ausserordentliche steuerliche Vergünstigungen, was auch für die Mitglieder vieler Konzernleitungen zutrifft. Bei der Standortsuche, sei es auch nur eine Erweiterung, werden ihnen Zugeständnisse gemacht, die einem Schweizer KMU nicht zugänglich sind. Die Unternehmen werden mit der Gesetzgebung im eigenen Land, soweit möglich, vor Forderungen aus dem Ausland geschützt. Bei Reisen von Regierungsmitgliedern ins Ausland sind mehr Vertreter der A-Schweiz anzutreffen als solche der B-Schweiz.

Weil die Steuerleistungen der Unternehmen der A-Schweiz berüchtigt tief sind, was zu Auseinandersetzungen zwischen kantonalen Steuerbehörden und der nationalen Finanzaufsicht geführt hat, tragen die Unternehmen der A-Schweiz kaum etwas bei zur Erhaltung der nationalen Infrastruktur. Die nationalstaatlichen Aufgaben werden von den Unternehmen der B-Schweiz und dem Schweizer Mittelstand, etwa dreissig Prozent der Bevölkerung, finanziert.

Die B-Schweiz, welche etwa 300000 Unternehmen und über 95\% der Bevölkerung ausmacht, klammert sich an den nationalen Mythos der erfolgreichen Schweiz, zu welcher auch die Firmen der A-Schweiz zählen. Das gilt jedoch nur beschränkt: Ein Alfred Schinder (Schindler Gruppe) ist ebenso eine Ausnahmefigur wie Dr. Christoph Blocher (Emser Werke) oder Peter Spuhler, der eine eigene Industrieholding aufgebaut hat. Trotz ihrer grossen Erfolge zählen sie nicht zur Weltklasse, wie dies für Nestlé, Novartis und Roche der Fall ist. Erst die kommenden Jahrzehnte werden zeigen, was von den letzten echten Schweizer Multis in Unabhängigkeit übrig bleiben wird. Der Fall Sika macht deutlich, dass mit jedem Erbgang das Risiko wächst, dass Schweizer Eigentümer aus der industriellen Verantwortung aussteigen, um in die Rolle des vermögenden Rentiers überzugehen. Das benachbarte Österreich hat diesen Status längst erreicht.

Das Gegenargument aller, welche die Bedeutung dieses Prozesses herabspielen möchten, lautet: „Auch unsere Schweizer Konzerne investieren im Ausland.“ Dabei wird vergessen, dass es gerade jene Nichtschweizer Konzerne sind, die ab Schweiz im Ausland investieren; der Anteil der KMU an diesem Prozess ist bedeutungslos.

In vielem, was schweizerisch genannt wird, ist wenig schweizerisches enthalten. Bisher hat dies dem Land vielen Wohlstand und wenigen grossen Reichtum gebracht. Ich meine, dieser Prozess ist im Rahmen der Globalisierung in vielem vorteilhaft und sollte fortgesetzt werden. Die damit verbundenen Kosten, darunter die Auflösung des Immunsystems der Schweiz und der schleichende Niedergang des hoch belasteten Mittelstands, sollten jedoch eine stärkere Beachtung finden. 
Klaus J. Stöhlker, Gründer der Stöhlker AG, Unternehmensberatung für Öffentlichkeitsarbeit, und Doyen der Schweizer PR-Branche.

Anschrift: Stöhlker AG, CH 8702, Zollikon, Zollikerstrasse 114, Tel.: +41(0)44 39648 88, E-Mail klaus.stoehlker\&stoehlker.ch 